\title{
Neuroscience Basis for Tactile Defensiveness and Tactile Discrimination among Children with Sensory Integrative Disorder
}

\author{
Aditi Srivastava* \\ 7 Frognal Place Sidcup Kent, London, United Kingdom \\ Submission: October 03, 2016; Published: November 30, 2016 \\ *Corresponding author: Aditi Srivastava, 7 Frognal Place Sidcup Kent, DA 14 6LR London, United Kingdom, Tel: 00-44-7446639157; \\ Email: shriaditi@gmail.com
}

\section{Case Blog}

Children with Sensory Integrative Dysfunction have difficulties in the processing and integrating sensory information. According to Miller et al. [1] around 5\%-15\% of children in the general population of kindergarten-age children demonstrate difficulties with sensory modulation. Moreover, a large number (80\%-90\% of children with Autism Spectrum Disorders demonstrate atypical sensory responsivity [2,3]. Sensory Integration is one of the most requested interventions by the parents of children with Aspergers, autism Spectrum Disorders $[4,5,6]$. It is the neurological process that organises sensation from one's own body and from the environment and makes it possible to use the body effectively within the environment. The sensory integration frame of reference was developed by Jean Ayres A [7,8], an occupational therapist (OT), with postdoctoral training in educational psychology and neuroscience.

Sensory integration (SI) is the information processing, [8]. It is the process by which the CNS develops and matures by organizing sensory information to produce an adaptive response [9]. Often children with neurological deficits such as Autism, Attention Deficit Hyperactivity Disorder (ADHD), Developmental Coordination Disorder (DCD), and Developmental Delay have difficulties in sensory integration and praxis. Some of the problems could be difficulties in finding objects from the bag without vision, manipulating writing and cutting tools in the classroom, high levels of distractibility and arousal levels. In sensory Integration theory, the tactile system is thought to be most relevant in regulating behavior [10]. Touch is the first sensory system to function in utero which mediates our first experiences in the world. We are much dependent on touch system during early life years until motor skills and cognitive abilities develop and specialise to guide our experiences and interactions.
The tactile system is one of the sensory systems which include nerves under the skin's surface that send information to the brain. This information includes light touch, pain, temperature, and pressure which play an important role in perceiving the environment as well as protective reactions for survival [11], and it is the physical barrier between us and the environment. It starts developing since 5 th week of pregnancy; supports a child to influence recognize different types of touch sensations as the child that grows. Functionally, this system supports in two important aspects, sucking and establishing emotional security. Touch sensations comfort baby in sucking, chewing and swallowing food.

Children who have difficulties in sucking may face challenges in eating different textures of food later in their lives. In other words, they might have oral hypersensitiveness (oral defensiveness). Emotional Security has major role every newborn's life since it establishes expressive protection, trust and acquaintance with mother or caregiver. It comprises of two primary functions known as protective and discriminative which will be discussed in details in this article. It has been concluded by researchers that protective and discriminative function should be considered separately.

\section{Discriminative System:}

The discriminative system is associated with the function of tactile discrimination such as detection of size, form, texture as well as movement across the skin [12]. The discriminative system is served by dorsal column-medial lemniscus (DCML) pathway, the receptors associated with DCML are responsible for responding to mechanical stimuli transmitting vibratory, touch pressure, discriminatory and deep pressure information [10]. Children displaying a cluster of behaviour such as being clumsy, messy, accident prone, dropping things from hand, having difficulties in managing buttons, zippers, laces and ties, 
difficulties in finding pencils from the bag without vision are hypothesised to have tactile discrimination difficulties. Moreover, these children lack normal awareness of being touched and position in space due to the poor tactile-proprioceptive system. They may demonstrate handwriting difficulties, poor drawing, colouring scissors and pasting skills.

According to Mailloux et al. [9], somatosensory system and praxis are directly linked. Children displaying low scores on Sensory Integration and Praxis Test, for tactile perception tests such as Finger Identification, Localization of Touch Stimuli, Graphesthesia, Manual Form Perception, in conjugation with Praxis tests, such as the Postural Praxis, Oral Praxis, Sequencing Praxis, Praxis on Verbal Command can be hypothesized to have Somatodyspraxia Mailloux et al. [9]. Somatodyspraxia is poor ability to plan and execute motor actions associated with signs of poor discrimination of touch and poor body scheme/ body awareness $[7,8]$. The receptors involved in DCML pathway transmit information from a periphery of the body to brain about vibration, touch-pressure, touch, proprioception and discrimination. Information ascends the DCML to two brain structures, the dorsal column of the spinal cord and the medial lemniscus in the brain stem [13].

The receptors involved in this process are Meissner's corpuscles found in glabrous skin within the dermis, rapidly adapting to mechanical stimuli. They are stimulated by slight pressure, and an action potential is generated. The generated action potential travels an axon and they continue inside the spinal cord, running up the posterior (dorsal) column [13]. The information from receptor site (skin) is transduced in the dorsal root ganglion of spinal cord (DRG) via axon. The generated action potential travels up to the cell body of the DRG of spinal cord ipsi-laterally. There is no synapse here and axons continue to pass the information within the posterior column of spinal cord, axons from the lower body continue to run in gracile tract however axons from upper body travel to cuneate tract (Level-T6) (i.e. middle thoracic and lower limbs of body).

The second order neurons cross over to the other side of medulla (internal arcurate) where it forms medial lemniscus; crossing over process is secondary decussation. At the medulla, the medial lemiscus ascends towards pons and mid brain (Brain stem) contra laterally and then projects in ventro-posterior lateral (VPL) nuclei of thalamus. From thalamus these fibers enter the somatosensory cortex forming the third order neurons. Within the primary and secondary motor cortices, S1 and S2 are areas of representation of DCML along with 5 and 7 areas of posterior parietal lobes. The projections of DCML, reaches somatosensory cortex S1, S2 as well as areas 5 and 7 of parietal lobe.

Children's poor tactile discrimination abilities can be reasoned due to damage in these areas which leads to poor functioning in fine manipulation skills [10]. suggested that S2 receives information from VPL and S1, S2. Hence S2 depends on upon sensory discrimination that occurs in $\mathrm{S} 2$ and projections from S2 to insular lobe are believed to be involved in tactile memory. Deficits in spatial perception, visual-motor integration and directed attention can be related to lesions in areas 5 and 7 of parietal lobe [10]. Damage in DCML system as well due to which sensory feedback to primary motor cortex is interrupted leading to deterioration of coordinated fine motor abilities of child. This can be evidenced by empirical research work done by Cohen (1999), who concluded that injury to DCML can lead to decreased sensory feedback to the cortex causing uncoordinated fine motor skills.

\section{Tactile Defensiveness}

Tactile defensiveness (TD) refers to a pattern of observable behavioral and emotional responses, which are aversive and negative to certain types of tactile stimuli that most people would find to be non-painful Royeen et al. [14]. Behaviorally these children in may pull themselves away on being touched lightly, may display distress by feel of new clothes, dislike tooth brushing, hair being washed. They may prefer to wear full sleeves shirt and avoid getting messy with finger paint, glue or tape. According to Ayres [6], tactile defensiveness is the response that occurs, when DCML system fails to exert inhibitory influence over Anterolateral System. Due to this child exhibits strong emotional response and escape-like behaviour and strong emotional response. In this condition non-standard neural messages are being sent to the motor cortex which in turn, overly stimulates the brain activity that is disorganised. This overstimulation can cause individual difficult to organise the behavior $[8,15]$.

The behavioural response system designed for protection and survival (AL System) predominated over a system (DCML System) designed to allow the organism respond to spatialtemporal qualities of the tactile stimuli (Ayres, 1972, p.215) [6]. Most of the AL pathways project into brain structures responsible for emotional tone and autonomic regulation, therefore, tactile defensive behaviours shown by children may be linked to correlations among these structures. It is composed of different pathways that function mainly to detect light touch, crude touch, pain and temperature which are protective in nature as opposed by DCML pathway which is discriminative in nature.

The pathways from the receptor site projections reach up to DRG. From here central processes from neurons reach the dorsal root of spinal cord. Many fibers reach the dorsal horn of the spinal cord called the tract of Lissauer. Most fibers synapse here with substantia gelatinosa, located in the dorsal horn of the Spinal Cord. These axons then decussate in the anterior white commissure of the spinal cord within several segments of their origin. After synapsing here most of neurons ascend in brain stem, VPL of Thalamus, Reticular Formation forming the Second orders neurons. These fibers from VPL of thalamus forming 


\section{Open Access Journal of Neurology \& Neurosurgery}

third order neurons terminate into the primary somatosensory cortex (Brodmann's areas 3, 1, 2 of the postcentral gyrus) into topologic manner.

These fibers also project to reticular system, preaqua-ductal area, which is, connected to limbic system via hypothalamus, hypothalamic and tectum areas. Projections to the tectum are associated with pain reception. VPL receives projections from DCML and AL and hence important center for interaction. However, it is assumed that DCML input is inhibited, due to which light touch is seen as aversive [10]. Children who display aversions on being touched, on their face or while hair washing as well as preference to stand in the end of line so as to avoid touch from other pupils, it may be reasoned due to tactile defensiveness.

The neurophysiological basis for this behaviour can be defined based on trigeminal pathway as it is responsible for carrying information on the face. The sensory information travels from face into trigeminal ganglion, axons project to the pons and spinal cord where they ascend and descend before synapsing Projections take place in sensory nucleus and in Trigeminal nerve, which carries pain, temperature and non-discriminative touch from face and mouth to the CNS. To conclude, the fibers project into ventral posterior lateral (VPL) nucleus and then primary somatosensory cortex where there is large presentation of mouth [10].

\section{References}

1. Ahn RR, Miller LJ, Milberger S, McIntosh DN (2004) Prevalence of parents' perceptions of sensory processing disorders among kindergarten children. Am J OccupTher 58(3): 287-293.

2. Rogers SJ, Ozonoff S (2005) Annotation: What do we know about sensory dysfunction in autism? A critical review of the empirical evidence. J Child Psychol Psychiatry 46(12): 1255-1268.
3. Tomchek SD, Dunn W (2007) Sensory processing in children with and without autism: A comparative study using the Short Sensory Profile. Am J Occup Ther 61(2): 190-200.

4. Green VA, Pituch KA, Itchon J, Choi A, O’Reilly M, et al. (2006) Internet survey of treatments used by parents of children with autism. Res Dev Disabil 27(1): 70-84.

5. Harrington JW, Rosen L, Garnecho A, Patrick, PA (2006) Parental perceptions and use of complementary and alternative medicine practices for children with autistic spectrum disorders in private practice. Journal of Developmental and Behavioral Pediatrics, 27(Suppl.), S156-S161.

6. Mandell DS, Novak MM, Levy S (2005) Frequency and correlates of treatment use among a community sample of children with autism. San Diego, CA. Proceedings of the 4th Annual International Meeting for Autism Research.

7. Ayres AJ (1972) Sensory integration and learning disorders. Western Psychological Services, Los Angeles, USA.

8. Ayres AJ (1989) Sensory integration and praxis tests manual. Western Psychological Services, Los Angeles, USA.

9. Mailloux Z, Mulligan S, Roley SS, Blanche E, Cermak S, et al. (2011) Verification and clarification of patterns of sensory integrative dysfunction. Am J OccupTher 65(2): 143-151.

10. Bundy AC, Fisher AG, Murray EA (2002) Sensory integration, theory and practice. ( $2^{\text {nd }}$ edn), FA Davis, Philadelphia, USA.

11. Hatch-Rasmussen (2013) What Is Sensory Integration? Therapy Northwest, PC Beaverton, OR 97005, USA.

12. Bundy AC, Fisher AG, Murray EA (1991) Sensory integration, theory and practice. ( $1^{\text {st }}$ edn), FA Davis, Philadelphia, USA.

13. Purves D, Augustine GJ, Fitzpatrick D (2001) last update, Central Pain Pathways: The Dorsal Column Leminscus Tract.

14. Royeen CB (1986) The development of a touch scale for measuring tactile defensiveness in children. Am J OccupTher 40(6): 414-419.

15. Lane SJ, Schaaf RC (2010) Examining the neuroscience evidence for sensory-driven neuroplasticity: implications for sensory-based occupational therapy in children and adolescents. Am J Occup Ther 64(3): 375-390.

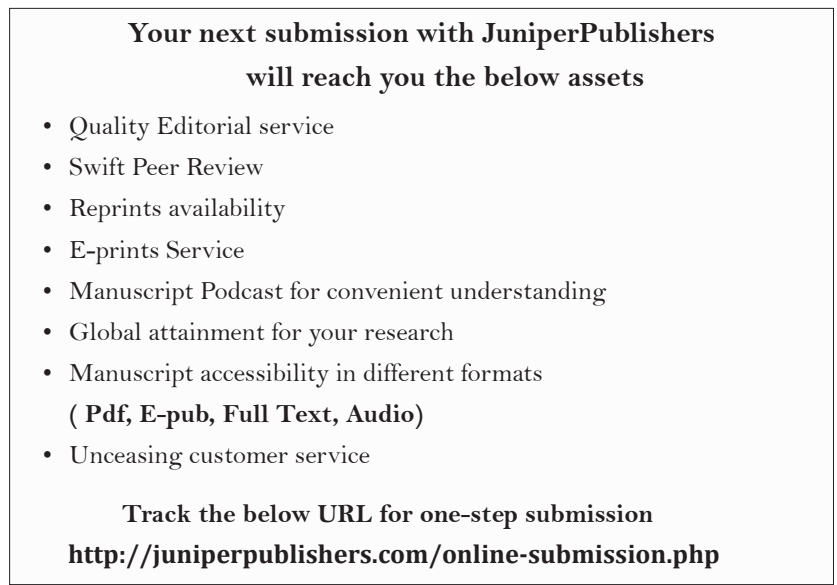

